\title{
THE JUMP IS DEFINABLE IN THE STRUCTURE OF THE DEGREES OF UNSOLVABILITY
}

\section{S. BARRY COOPER}

Recursion theory deals with computability on the natural numbers. A function $f$ from $\mathbf{N}$ to $\mathbf{N}$ is computable (or recursive) if it can be calculated by some program on a Turing machine, or equivalently on any other general purpose computer. A major topic of interest, introduced in Post [23], is the notion of relative difficulty of computation. A function $f$ is computable relative to a function $g$ if after equipping the machine with a black box subroutine that provides the values of $g$, there is a program (which now may call $g$ via the subroutine) which computes $f$. In this case we write $f \leq_{T} g$. Two functions are Turing equivalent if each is computable relative to the other; the equivalence classes are called Turing degrees. These degrees form a partial ordering $\mathscr{D}$ under the induced reducibility relation $\leq$. The structural analysis of the partial ordering $\mathscr{D}$ has been a major area of research in recursion theory since the pioneering paper of Kleene and Post [14].

Kleene and Post proved a number of results on the structure of $\mathscr{D}$ including the embeddability of arbitrary countable partial orders into $\mathscr{D}$, and obtained partial results on extendability of a given embedding to a larger domain. This line of investigation was pursued by many people over the next twenty-five years, culminating in essentially complete solutions of these problems, and a characterization of the possible ideals of the structure $\mathscr{D}$ (see Lachlan and Lebeuf [16] and Lerman [17], [18]).

Kleene and Post also considered the enriched structure $\mathscr{D}^{\prime}$ equipped with the "jump operator", denoted ', which is a canonical operation on degrees which takes each degree $\mathbf{d}$ to a strictly

Received by the editors September 22, 1989 and, in revised form, November 13, 1989.

1980 Mathematics Subject Classification (1985 Revision). Primary 03D30.

Research supported by a C.N.R. visiting professorship while the author was at the University of Siena, March-June, 1989. We would also like to gratefully acknowledge the support and hospitality of the members of the Department of Mathematics at the University of Siena. 
larger degree $d^{\prime}$. The importance of the jump $d^{\prime}$ of a degree d derives from its definition as the largest degree containing sets which are effectively enumerable using an oracle chosen from d (where we locate the degree of a set via the identification of sets with their characteristic functions). More generally, Post's theorem [23] (that $X \in \Delta_{n+1}^{A} \leftrightarrow X \leq_{T} A^{(n)}$ ) relates the quantifier forms of many naturally occurring sets of numbers to the ascending sequence $\mathbf{0}<\mathbf{0}^{\prime}<\mathbf{0}^{\prime \prime}<\cdots<\mathbf{0}^{(n+1)}=\left(0^{(n)}\right)^{\prime}<\cdots \quad(\mathbf{0}$ being the degree of the recursive sets). Because of this connection, this jumpderived sequence plays an essential role in calculating bounds on complexity as in bounds for embedding problems in Kleene-Post [14] onwards.

The past twelve years or so have produced major progress on more global questions about both $\mathscr{D}$ and $\mathscr{D}^{\prime}$ concerning automorphisms and definability in these structures. Jockusch and Simpson [12] provided the first major definability results (defining many natural classes of degrees in $\mathscr{D}^{\prime}$ ) so initiating a string of successes in this area (amongst others [7, 9, 19, 20, 29-31]). Most of these definability results have built on earlier work of a number of people on embeddings as initial segments of the degrees and codings into the degrees (for example $[13,16,17,24]$ ), although the simpler coding technique of Slaman and Woodin [33] suffices to prove all previously known global results concerning automorphisms and definability for $\mathscr{D}$ (see section V.7 of [21]). A new element was provided by Jockusch and Shore $[10,11]$ who used their own notion of pseudo jump in a very productive way to obtain a number of results, including the definability in $\mathscr{D}$ of the set of arithmetical degrees (those degrees below $\mathbf{0}^{(n)}$ some $n \geq 0$ ). Unremitting refinement of such techniques (particularly coding techniques) have culminated [26] in the first-order definability of $0^{(3)}$ in $\mathscr{D}\left(\leq 0^{\prime}\right)$ and [28] in the definability of all jump classes $\mathbf{H i g h}_{n}$, Low ${ }_{n}$ of degrees $\leq \mathbf{0}^{\prime}$ in $\mathscr{D}\left(\leq \mathbf{0}^{\prime}\right)$ for each $n \geq 3$.

The most fundamental definability problem was raised by Kleene and Post themselves when they speculated about the possibility that the jump operator is intrinsically determined by the structure $\mathscr{D}$. Our main result answers this question: The jump operator is definable in terms of the partial ordering $\mathscr{D}$; and, in fact, so is the notion of "recursively enumerable in". This result immediately allows one to improve many previous theorems on automorphisms, homogeneity, and more general definability ques- 
tions, some of which we list below.

Our solution to this problem lies largely outside of the line of development which started with the Jockusch and Simpson paper (coding plays no part) but does use elements of the Jockusch and Shore $n$-REA operator machinery, and in general outline follows the plan for the definability of the degrees of the arithmetic sets in [11]. The main ingredient in this global result is a theorem of local degree theory which is in direct descent from the Lachlan [15] monster construction (as refined and re-presented by Harrington, Slaman, Soare and others). It also benefits from the steady development over the years (recently see for example [2, 4, 27, 32]) of the techniques of $\Delta_{2}$-degree theory, and the recent renewed interest in the $d$-r.e. degrees (see [1, 5 and 6]). [18, 21 and 34] provide further information on these topics.

Definition. A set $D$ is $d$-recursively enumerable $(d$-r.e.) if and only if $D=A-B$, some r.e. $A, B$. d is $d$-r.e. if and only if it contains a $d$-r.e. set $D$.

Definition. $\mathbf{d}$ is unsplittable over $\mathbf{a}$ avoiding $\mathbf{b}$ if and only if $\mathbf{a}, \mathbf{b} \leq \mathbf{d}, \mathbf{b} \not \mathbf{a}$, and for every $\mathbf{d}_{0}, \mathbf{d}_{1}<\mathbf{d}$, if $\mathbf{a}<\mathbf{d}_{0}, \mathbf{d}_{1}$ then either $\mathbf{b} \leq \mathbf{d}_{0}$ or $\mathbf{d}_{1}$, or $\mathbf{d} \neq \mathbf{d}_{0} \cup \mathbf{d}_{1}$. We say $\mathbf{d}$ is relatively unsplittable if and only if $\mathbf{d}$ is unsplittable over $\mathbf{a}$ avoiding $\mathbf{b}$ some a, b .

Theorem 1. There exists a relatively unsplittable $d$-r.e. degree.

Corollary 1. There is a cone of relatively unsplittable degrees with base degree $\mathbf{0}^{\prime \prime}$.

Proof. Relativising the proof of the theorem to a given set $B$, we can obtain a 2-REA operator $J$ such that $J(B)=B \oplus$ $\left(W_{c_{1}}^{B}-W_{c_{0}}^{B}\right)$ for appropriate $c_{0}, c_{1}$, and $\operatorname{deg}(J(B))$ is unsplittable over a degree $a \geq \operatorname{deg}(B)$. We can then apply Theorem 2.3 of [11] as in Corollary 2.4 of the same paper to obtain the result.

Corollary 2. $\mathbf{0}^{\prime}$ is definable in the degrees as the largest degree satisfying

$\neg(\exists \mathbf{a}, \mathbf{b})(\mathbf{x} \cup \mathbf{a}$ is unsplittable over $\mathbf{a}$ avoiding $\mathbf{b})$.

Proof. For each r.e. $\mathbf{x}$ and each $\mathbf{a}, \mathbf{x} \cup \mathbf{a}$ is r.e. in $\mathbf{a}$, so if $\mathbf{x} \cup \mathbf{a} \geq \mathbf{b}$ and $\mathbf{b} \$ \mathbf{a}$ the relativised Sacks splitting theorem (see p. 124 of [34]) gives us a splitting of $\mathbf{x} \cup \mathbf{a}$ over a avoiding $\mathbf{b}$. On the other hand, applying Posner/Robinson cupping [22], essentially in the 
form presented by Jockusch and Shore in Theorem 3.2 of [11], ${ }^{2}$ for each $D \geq_{T} \varnothing^{\prime \prime} \oplus X$ with $X{⿱_{T}}_{T} \varnothing^{\prime}$ we can find an $A$ such that $X \oplus A \equiv_{T} D \equiv_{T} J(A)$, where $J$ is the 2-REA operator of Corollary 1 . The corollary follows immediately. Post:

We now get, answering the question first posed by Kleene and

Corollary 3. The Turing jump is definable in the structure of the degrees of unsolvability.

Proof. Relativising Corollary 2, $\mathbf{a}^{\prime}$ is definable in $\mathscr{D}(\geq \mathbf{a})$.

Since we can now define all the degrees $\mathbf{0}^{(n)}, n \in \omega$, and the set of degrees below $\mathbf{0}^{\prime}$, we immediately get the following, complementing Shore [28]:

Corollary 4. All the jump classes $\mathrm{High}_{n}$ and $\mathrm{Low}_{n}, n>0$, are definable in $\mathscr{D}$.

Of course all other classes definable in terms of the jump (without parameters) are now definable in $\mathscr{D}$, including those of the generalized high and low hierarchies. The set of arithmetical degrees (defined in $\mathscr{D}$ by Jockusch and Shore in [11]) is now trivially definable as the least jump ideal (using Spector's exact pair theorem). The problem of completely characterizing the definable jump classes of the form $\left\{\mathbf{x} \mid \mathbf{x}^{\prime}=\mathbf{c}\right\}$, $\mathbf{c}$ an arithmetical degree $\geq \mathbf{0}^{\prime}$, remains open (however see footnote 3 below).

Corollary 3 also provides alternative proofs of results concerning $\mathscr{D}$ where a significantly different proof of the analagous result for $\mathscr{D}^{\prime}$ exists. For example Feiner's solution [8] of the strong homogeneity problem (or the Yates proof [35] presented in Lerman [18]) now gives a solution to the homogeneity problem (as originally stated in Rogers [25]).

We can also improve results concerning $\mathscr{D}$ where stronger versions for $\mathscr{D}^{\prime}$ are already available. For example:

Corollary 5. If $f$ is an automorphism of $\mathscr{D}$ then $f(\mathbf{a})=\mathbf{a}$ for all $\mathbf{a} \geq \mathbf{0}^{(3)}$.

Proof. Richter [24] showed that each jump preserving automorphism of the degrees fixes all $\mathbf{a} \geq 0^{(3)}$.

${ }^{2}$ Although the join theorem is not known for arbitrary REA operators, we can apply the special cupping derivable for operators of the particular form got from $n$-r.e. operators. We are grateful to C. G. Jockusch and R. A. Shore for providing us with helpful technical comments concerning this. 
Corollary 6. ${ }^{3}$ If $\mathscr{D} \equiv \mathscr{D}(\geq \mathbf{b})$ then $\mathbf{b}^{(3)}=\mathbf{0}^{(3)}$.

Proof. Use Theorem 4.4 of [26].

To prove the theorem, we construct a $d$-r.e. set $D$ and sets $A, B \leq_{T} D$ satisfying the requirements:

$$
\begin{gathered}
P_{k}: B \neq \Theta_{k}^{A}, \\
Q_{k}: D=\Psi_{k}\left(\Phi_{k}^{D}, \widehat{\Phi}_{k}^{D}\right) \rightarrow B=\Gamma_{k}\left(\Phi_{k}^{D}, A\right) \vee B=\Lambda_{k}\left(\widehat{\Phi}_{k}^{D}, A\right),
\end{gathered}
$$

where $\left(\Theta_{k}, \Psi_{k}, \Phi_{k}, \widehat{\Phi}_{k}\right)$ is some standard listing of the quadruples of p.r. functionals and $\Gamma_{k}, \Lambda_{k}$ are p.r. functionals to be constructed. We also have an overall constraint that $A=\Omega^{D}$, $B=\widehat{\Omega}^{D}, \Omega, \widehat{\Omega}$ to be defined during the construction.

The basic module for a pair $P, Q$ of requirements (dropping the subscripts) is reminiscent of Lachlan's nonsplitting theorem. Roughly speaking, as in the monster, we monitor $l\left(D, \Psi\left(\Phi^{D}, \widehat{\Phi}^{D}\right)\right)$ and try first to implement the $\Gamma$-strategy, using Harrington's “capricious destruction" to try to produce a situation where we can satisfy $B(x) \neq \Theta^{A}(x)$, say, by changing $B(x)$ and restraining $A\lceil\theta(x)$. And then failing this using "honestification" to try and produce a $\Phi^{D}$-permission via $\Gamma$ for $x \searrow B$ without an $A\lceil\theta(x)$ change (using the equation $D=\Psi\left(\Phi^{D}, \widehat{\Phi}^{D}\right)$ ). And finally, switching to the $\Lambda$-strategy when all else fails, the $\Lambda$-strategy mirroring the $\Gamma$-strategy but working within the more favorable framework provided by the failure of the $\Gamma$-strategy. However, the fact that we are dealing with $\Phi^{D}, \widehat{\Phi}^{D}$ which are not r.e. and over which we have only limited control, means we must take special care in producing the $\Phi^{D}$ - or $\widehat{\Phi}^{D}$-permissions (using extended honestification and the facility for making $D$ - and $A$-changes independently of $B)$, and must be prepared to use the $d$-r.e.ness of $D$ to save our $\Gamma$ - or $\Lambda$-strategies when conflict between lower priority $P$ requirements results in such permissions disappearing. We will be able to live with $\Phi^{D}$ or $\widehat{\Phi}^{D}$ volatility, except when it reaches a point at which it results in a breakdown in $D=\Psi\left(\Phi^{D}, \widehat{\Phi}^{D}\right)$ in which case $Q$ is satisfied. The framework for reconciling the demands of the strategies for different pairs of requirements is similar to that of [3].

\footnotetext{
${ }^{3}$ In a similar vein to Corollaries 5 and 6 , Odifreddi points out that every relation on degrees above $\boldsymbol{0}^{(3)}$ is definable in $\mathscr{D}$ if and only if it is definable in second-order arithmetic. Consequently (as Jockusch and Shore observe) if $\mathbf{c} \geq \mathbf{0}^{(3)}$ is arithmetic then, using the definability of the jump, $\left\{\mathbf{x} \mid \mathbf{x}^{\prime}=\mathbf{c}\right\}$ is definable.
} 
Theorem 2. If $\mathbf{d}<\mathbf{0}^{\prime}$ is not $\mathbf{r}$. e., there exist $\mathbf{a}, \mathbf{b}<\mathbf{0}^{\prime}$ such that $\mathbf{a} \cup \mathbf{d}$ is unsplittable over $\mathbf{a}$ avoiding $\mathbf{b}$.

Corollary 7. The recursively enumerable degrees are definable in $\mathscr{D}\left(\leq \mathbf{0}^{\prime}\right)$, and hence in $\mathscr{D}$.

Proof. If $\mathbf{d} \leq \mathbf{0}^{\prime}$, d is r.e. $\leftrightarrow\left(\forall \mathbf{a}, \mathbf{b} \leq \mathbf{0}^{\prime}\right) \quad(\mathbf{a} \cup \mathbf{d}$ is not unsplittable over a avoiding b). Since $\mathbf{0}^{\prime}$ is definable in $\mathscr{D}$, the result follows.

Corollary 8. The relation " $\mathbf{d}$ is $\mathbf{b}-\mathrm{REA}$ " is definable in $\mathscr{D}$.

Proof. d is $\mathbf{b}-\mathrm{REA} \leftrightarrow \mathbf{d} \in\left[\mathbf{b}, \mathbf{b}^{\prime}\right]$ and $\left(\forall \mathbf{a}, \mathbf{c} \in\left[\mathbf{b}, \mathbf{b}^{\prime}\right]\right)(\mathbf{a} \cup \mathbf{d}$ is not unsplittable over a avoiding $\mathbf{c}$ ). Since the jump is definable in the degrees, the result follows.

The requirements for Theorem 2 take the form:

$$
\begin{gathered}
P_{k}: B \neq \Theta_{k}^{A} \vee\left(\exists A^{*} \text { co-r.e. }\right)\left(A^{*} \equiv_{T} D\right), \\
Q_{k}: D=\Psi_{k}\left(\Phi_{k}^{A, D}\right) \rightarrow B=\Gamma_{k}\left(\Phi_{k}^{A, D}, A\right) \vee B=\Lambda_{k}\left(\widehat{\Phi}_{k}^{A, D}, A\right),
\end{gathered}
$$

where $D$ is now given and we have an overall constraint $B=$ $\Omega^{A, D}$.

There are many elements of the earlier proof in the construction for Theorem 2, but $D$ now leads with changes rather than us having control over them. We lean heavily on the $A^{*} \equiv_{T} D$ pseudo-outcome, using $\Phi_{k}^{A, D}, \widehat{\Phi}_{k}^{A, D}$ and $A$ in producing "positive" $A$-traces for elements of $B$ which involve extractions from $A^{*}$ in signalling $B$-changes.

We notice that with some care in how we define $A$ in the case $D$ is $d$-r.e. we can obtain Theorem 1 as a special case of Theorem 2.

\section{REFERENCES}

1. M. M. Arslanov, Structural properties of the degrees below $\mathbf{0}^{\prime}$, Dokl. Akad. Nauk. SSSR, (new series) 283 (2) (1985), 270-273.

2. S. B. Cooper, The strong anticupping property for recursively enumerable degrees, J. Symbolic Logic 54 (1989), 527-539.

3. _ـ A jump class of noncappable degrees, J. Symbolic Logic 54 (1989), 324-353.

4. S. B. Cooper and R. L. Epstein, Complementing below recursively enumerable degrees, Ann. Pure Appl. Logic 34 (1987), 15-32.

5. S. B. Cooper, S. Lempp, and P. Watson, Weak density and cupping in the d-r.e. degrees, Israel J. Math. 67 (1989), 137-152.

6. S. B. Cooper, L. Harrington, A. H. Lachlan, S. Lempp, and R. I. Soare, The d-r.e. degrees are not dense (in preparation). 
7. R. L. Epstein, Degrees of unsolvability: Structure and theory, Lecture Notes in Mathematics No. 759, Springer-Verlag, Berlin, Heidelberg, New York, 1979.

8. L. Feiner, The strong homogeneity conjecture, J. Symbolic Logic 35 (1970), 375-377.

9. L. Harrington and R. A. Shore, Definable degrees and automorphisms of $\mathscr{D}$, Bull. Amer. Math. Soc. (new series) 4 (1981), 97-100.

10. C. G. Jockusch, Jr. and R. A. Shore, Pseudo jump operators I: The R.E. case, Trans. Amer. Math. Soc. 275 (1983), 599-609.

11. __ Pseudo jump operators II: Transfinite iterations, hierarchies, and minimal covers, J. Symbolic Logic 49 (1984), 1205-1236.

12. C. G. Jockusch, Jr. and S. G. Simpson, A degree theoretic definition of the ramified analytic hierarchy, Ann. Math. Logic 10 (1976), 1-32.

13. C. G. Jockusch, Jr. and R. M. Solovay, Fixed points of jump preserving automorphisms of degrees, Israel J. Math. 26 (1977), 91-94.

14. S. C. Kleene and E. L. Post, The upper semi-lattice of degrees of recursive unsolvability, Ann. of Math. (2) 59 (1954), 379-407.

15. A. H. Lachlan, $A$ recursively enumerable degree which will not split over all lesser ones, Ann. Math. Logic 9 (1975), 307-365.

16. A. H. Lachlan and R. Lebeuf, Countable initial segments of the degrees of unsolvability, J. Symbolic Logic 41 (1976), 289-300.

17. M. Lerman, Initial segments of the degrees of unsolvability, Ann. of Math. (2) 93 (1971), 365-389.

18. _ Degrees of unsolvability, Springer-Verlag, Berlin, Heidelberg, New York City, Tokyo, 1983.

19. A. Nerode and R. A. Shore, Second order logic and first order theories of reducibility orderings, The Kleene Symposium (J. Barwise et al., eds.), North-Holland, Amsterdam, 1980, pp. 181-200.

20. _ Reducibility orderings: theories, definability and automorphisms, Ann. Math. Logic 18 (1980), 61-89.

21. P. Odifreddi, Classical recursion theory, North-Holland, Amsterdam, New York, Oxford, 1989.

22. D. Posner and R. W. Robinson, Degrees joining to $\mathbf{0}^{\prime}$, J. Symbolic Logic 46 (1981), 714-722.

23. E. L.Post, Recursively enumerable sets of positive integers and their decision problems, Bull. Amer. Math. Soc. 50 (1944), 284-316.

24. L. J. Richter, On automorphisms of the degrees that preserve jumps, Israel J. Math. 32 (1979), 27-31.

25. H. Rogers, Jr., Theory of recursive functions and effective computability, McGraw-Hill, New York, 1967.

26. R. A. Shore, On homogeneity and definability in the first order theory of the Turing degrees, J. Symbolic Logic 47 (1982), 8-16.

27. _ A non-inversion theorem for the jump operator, Ann. Pure Appl. Logic 40 (1988), 277-303.

28. __ Defining jump classes in the degrees below $0^{\prime}$, Proc. Amer. Math. Soc. 104 (1988), 287-292.

29. S. G. Simpson, First order theory of the degrees of unsolvability, Ann. of Math. (2) 105 (1977), 121-139. 
30. T. A. Slaman and R. A. Shore, Working below a $\operatorname{low}_{2}$ recursively enumerable degree (to appear).

31. __ Working below a high recursively enumerable degree (in preparation).

32. T. A. Slaman and J. Steel, Complementation in the Turing degrees, J. Symbolic Logic 54 (1989), 160-176.

33. T. A. Slaman and W. H. Woodin, Definability in the Turing degrees, Illinois J. Math. 30 (1986), 320-334.

34. R. I. Soare, Recursively enumerable sets and degrees, Springer-Verlag, Berlin, Heidelberg, New York, London, 1987.

35. C. E. M. Yates, Initial segments and implications for the structure of degrees, Conference in Mathematical Logic, London, 1970 (W. Hodges, ed.), Springer-Verlag, Berlin, Heidelberg, New York, 1972.

School of Mathematics, University of LeEds, Leeds LS2 9JT, ENGLAND 